\title{
AN EPISTEMOLOGY FOR THE PLATONIST? \\ PLATONISM, FIELD'S DILEMMA, AND JUDGMENT-DEPENDENT TRUTH
}

\author{
Tommaso PIAZZA* \\ University of Porto
}

\begin{abstract}
Summary
According to Hartry Field, the mathematical Platonist is hostage of a dilemma. Faced with the request of explaining the mathematicians' reliability, one option could be to maintain that the mathematicians are reliably responsive to a realm populated with mathematical entities; alternatively, one might try to contend that the mathematical realm conceptually depends on, and for this reason is reliably reflected by, the mathematicians' (best) opinions; however, both alternatives are actually unavailable to the Platonist: the first one because it is in tension with the idea that mathematical entities are causally ineffective, the second one because it is in tension with the suggestion that mathematical entities are mind-independent. John Divers and Alexander Miller have tried to reject the conclusion of this argument-according to which Platonism is inconsistent with a satisfactory epistemology for arithmetic — by re-describing the second horn of the dilemma in light of Crispin Wright's notion of judgment-dependent truth; in particular they have contended that once arithmetical truth is conceived in this way the Platonist can have a substantial epistemology which does not conflict with the idea that the mathematical entities exist mind-independently. In this paper I analyze Wright's notion of judgment-dependent truth, and reject Divers and Miller's argument for the conclusion that arithmetical truth can be so characterized. In the final part, I address the worry that my argument generalizes very quickly to the conclusion that no area of discourse could be characterized as judgment-dependent. As against this conclusion, I indicate under what conditions-notably not satisfied in Divers and Miller's case, but possibly satisfied in others-a discourse's judgment-dependency can be successfully vindicated.
\end{abstract}

\footnotetext{
* Researcher C2007, co-financed by FSE and POPH.
} 
Introduction: the Euthyphro contrast, and Wright's notion of judgment-dependent truth

According to C. Wright, the satisfaction of the Principle of Knowability (PK) - according to which $p$ entails that it is possible to know that $p$-is not sufficient to distinguish between areas of discourse deserving an antirealist interpretation and those which deserve a realist interpretation. Contra M. Dummett, who has championed the opposite view, Wright suggests that more must be added to vindicate antirealism concerning some debated topic than simply showing that the relevant discourse is subject to PK.

Suppose in fact that every true statement expressible within a discourse is such that, under the appropriate circumstances of epistemic optimality, it is judged to be so, and vice versa, that any judgment issued in conditions of epistemic optimality is true. The bi-conditional which expresses this relation:

$$
\text { (EC) } p \leftrightarrow(\mathrm{C} \rightarrow \mathrm{J} p)
$$

(where $\mathrm{C}$ specifies the conditions of epistemic optimality relative to the range of statements over which $p$ varies, and J is to be read as "it is judged that"), entails the satisfaction of $\mathrm{PK}^{1}$, yet it still leaves underdetermined whether we should attach explanatory priority to its right-hand side, or to its left-hand side. Much in a realistic spirit, in fact, one could hold that the

1. If every true statement in a discourse is such that, under the relevant optimal epistemic conditions, it is judged to be true, satisfaction of PK follows from the natural assumptions that (a) knowledge is justified true belief, that (b) the epistemic conditions mentioned in the righthand side of the bi-conditional are such as to confer justification on the opinions they enable, that (c) these conditions are possibly instantiated, and that (d) if the antecedent of a conditional is possible, so is its consequent. Suppose in fact that a discourse D sustains the relevant bi-conditional: it follows, by (a), (b), and (c) that there are possible circumstances under which every truth in the discourse is known. By (d), it then follows that every truth is possibly known. To be noted, premise (a) does not ignore the familiar counterexamples proposed by Gettier against the standard definition of knowledge; rather, it is proposed on the assumption that the "optimal epistemic conditions" referred to within the bi-conditional are such as to escape usual Gettierization. Admittedly, much more could be said to support (a); however, it seems sensible to suppose that whatever additional material is needed to escape Gettier-like counterexamples, it must be already in place if the conditions under which every statement is assessed are to count as "optimal". Finally, to call into question the sense of possibility referred to within (c) would be of no help in resisting the entailment. For whatever sense of possibility one has in mind when requiring those epistemic conditions to be possibly instantiated must obviously be the same one has in mind when advocating the principle that every truth is possibly known. 
reason why every truth in the discourse would be judged if the epistemic conditions were good enough is that, under those conditions, we would be best suited to detect the facts in that area, to the extent that not even a single fact could remain unstated; on the opposite direction, however, one could hold that matters are the other way around: one could oppose the detectivist view, advanced by the realist, and maintain that being judged in conditions of epistemic optimality is the conceptual ground of truth in the discourse.

This opposition between the realist and the antirealist gives rise to what Wright—echoing Plato's dialogue—has dubbed the Euthyphro Contrast: according to Socrates - the realist character-it is a merit of the alleged conditions of epistemic optimality that they put us in a position to know everything there is to know within a given area; according to Euthyphrothe antirealist character-judgment in conditions of epistemic optimality is the raison d'etre of truth in the discourse.

To give content to this opposition Wright has cheerfully described a recipe for resolving the dispute, by devising a range of conditions which a discourse subject to EC will have to meet if, intuitively, it is to deserve (or fail to deserve) a realist interpretation ${ }^{2}$. The first one is a meta-condition: when specifying the debated bi-conditional, for $p$ ranging over the statements expressible within some specific discourse, the relevant $\mathrm{C}$-conditions must be specified substantially. In a word, this means that such conditions do not have to make the principle itself trivially true, but must be specified in light of a "constructive epistemology" for the statements in question. Suppose in fact that, for $p$ ranging over ascriptions of colour to middlesized perceptual objects, one specified EC as follows:

$\left(\mathrm{EC}^{*}\right) p \leftrightarrow$ (the epistemic conditions are good enough to guarantee that if $p$ is true then it is judged that $p \rightarrow \mathrm{J} p$ ).

As it is clear, EC* fails to tell us something informative concerning the conditions which would guarantee-one way or another-that every colour ascription would be true, and that every colour a thing possesses

2. Wright's discussion of the Euthyphro Contrast initially concentrates on bi-conditionals like (EC) $(1992,108)$; however, in view of the potential difficulty that the obtaining of $C$ could interfere with the truth-value of $p$, and so engender the so-called conditional fallacy, he ends up proposing provisional equations like the following: $\mathrm{C} \rightarrow(p \leftrightarrow$ it is judged that $p$ ) (1992, 119). Nothing significant, in the present paper, hangs on whether we choose either formulation; therefore I decided to stick to the initial one conveyed by EC. 
would be ascribed. However, this is not its gravest shortcoming. To see why will help locate the proper function of the second condition envisaged by Wright.

Suppose that the Socratic theorist is right, and that the satisfaction of the $\mathrm{C}$-conditions indeed puts us in a position to detect every truth expressible within D. In this case, it seems sensible to suppose that this fact must hold contingently, and that we can come to know this fact only a posteriori, upon assessing our own cognitive undertakings with respect to the D-domain. In fact, it seems conceivable that our own epistemic set-up, or the features of our epistemic play-ground might have been otherwise than they are; so it is natural to suppose that, if the proper determination of a set of truths is conceptually independent of our investigative practice, the conditions under which we are (or would be) best placed to detect a given body of truths might have failed to be so ${ }^{3}$. On the other hand, suppose that it is the Euthyphronist who is right, and that the $\mathrm{D}$-truths are merely constituted by the opinions delivered under the C-conditions. In this case it is sensible to suppose that this fact must hold necessarily, and that we can come to know it a priori, by reflecting on the conceptual link between best opinion and truth in the discourse. If that much is conceded, the reason why we should be willing to enforce the requirement that the $\mathrm{C}$-conditions must be specified substantially, i.e. in a way which does not render the relevant instance of EC trivially true, is that every unsubstantial instance, like EC* above, is both necessarily true, and knowable a priori, and so such as to prevent by definition the desired modal and epistemological opposition from arising. In a word, the C-conditions must be specified so as to leave it open that the relevant instance of EC may turn out to be contingent and a posteriori.

A third condition ${ }^{4}$ proposed by Wright to adjudicate the Euthyphro Contrast about a given area of discourse $\mathrm{D}$ requires that the $\mathrm{C}$-conditions

3. "[...] [I]f, as according to Socrates' detectivist view, best opinion is merely responsive to truth, then it seems that it ought to be a possibility that the causal order be so constituted that the opinions formed under the conditions which, as things are, ensure that they track the facts, might not have done so." (Wright 1992, 112)

4. Wright also proposes as a fourth condition the Extremal Condition. This condition has the function of discriminating among "recognizably a priori true, substantially specified [EC-instances] whose C-conditions avoid all use of the distinctive concepts of the discourse" $(1992,123)$ which sustain an Euthyphronic interpretation from those which sustain the (Socratic) suspect that the range of facts with which we are dealing, though conceptually independent of our cognitive activity, is one in which simply "there is, a priori, no possibility of their misrepresentation by best opinion" (ibidem). The idea is that the satisfaction of the first three conditions-substantiality, 
mentioned by the relevant instance of EC, if its Euthyphronic interpretation has to be vindicated, be specified independently of the presupposition that some D-truths have already determined extensions. Suppose in fact that, when trying to assess the status of a given instance of EC, one specified the conditions of epistemic optimality associated to colour ascriptions by requiring that an object be stable in colour throughout a period of observation for the opinion thereby delivered to count as best. Clearly, in this case the conditions which have to be satisfied if an opinion to the effect that a given object is red is to count as best must already include that the object has instantiated, throughout the relevant period of observation, one single chromatic property. However, according to the Euthyphronist it is the very truth value of this kind of statements-colour ascriptions - that the opinion delivered under the conditions of epistemic optimality so described must determine. If one nonetheless pleads for an Euthyphronic interpretation of the relevant instance of EC, clearly inconsistency threatens $s^{5}$, so that the upshot of the irreducible presence of statements in the discourse whose truth-value is logically antecedent to the very satisfaction of the associated C-conditions must inescapably be the vindication of its Socratic interpretation. It follows that the satisfaction of the independence condition constitutes a necessary condition for establishing the Euthyphronic interpretation, and that failure to specify the C-conditions associated to a discourse independently of the truth of some statements expressible within it constitutes a sufficient condition for accepting the realist interpretation endorsed by the Socratic theorist.

This rough presentation of the Euthyphro Contrast should suffice to illustrate Wright's notion of judgment-dependent truth in terms of the following principle:

necessity and a priori knowability, and independence - may to some extent leave undetermined whether the best opinions in a discourse are guaranteed a priori to track the facts not as a result of the conceptual implication of human responses in the determination of their extensions, but rather as a result of some general feature of the corresponding truth-conferring states of affairs.

5. The inconsistency at issue is not one of circularly characterizing the truth-conditions of $p$ (left-hand side of EC) in terms of the opinions delivered in epistemic conditions whose specification requires the use of $p$ itself (right-hand side of EC). I agree with Wright, who explicitly maintains that EC-instances involving C-conditions specified less-than-independently, since they are not proposed as reductive analyses, do not have to face this specific objection from circularity. Rather, the inconsistency at issue is one of maintaining that truth in a discourse, for instance the chromatic one, is constrained by best opinion while at the same time explaining what makes opinion best by "implicitly presupposing some mode of constitution of color facts which is conceptually unconstrained by best opinion and hence potentially at odds with the Euthyphronist's central claim” (1992, 121). 
(JD-T) The truth-predicate finding application within a discourse A is judgment-dependent iff, for $p$ ranging over A-statements, a substantial and independent specification of the associated conditions of epistemic optimality gives rise to a necessary and a priori true instance of EC (whose explanation cannot proceed by invoking the special nature of the implicated truthconferring states-of-affairs).

In this paper I shall not be primarily concerned with JD-T; rather, I shall address a recent proposal, advanced by J. Divers and A. Miller, which puts this principle at the service of rescuing Arithmetical Platonism from a recent challenge, advanced by $\mathrm{H}$. Field, to explain the mathematicians' reliability as knowledge-gatherer consistently with the claim that mathematical objects are abstract, and mind-independent. Along with the authors' suggestion, the Platonist can successfully meet Field's challenge by contending that arithmetical truth is judgment-dependent, much in the sense conveyed by JD-T, and by arguing that this supposition still makes room for the idea that, under a certain respect, mathematical objects do exist independently of the mind. Elsewhere (Piazza 2009) I have suggested that the latter claim - that arithmetical truth can be coherently thought to be judgment-dependent, and nonetheless to concern a mind-independent realm of entities — can be made to work only against a notion of mind-independent existence which is philosophically dubious and, moreover, hardly relevant to the realism/antirealism issue. However, it is undeniable that Divers and Miller's position would still deserve a great deal of interest, were it possible to show that it is the only form of Platonism - no matter how weak, and how unsatisfactory the notion of mind-independent existence upon which it is based-to which a sound epistemology, consistent with Field's challenge, could be made available. And it is also undeniable that, were it possible to show that arithmetical truth is judgment-dependent, this desideratum could be made available quite straightforwardly: if arithmetical truth is constitutively dependent on what mathematicians would judge, were they to operate in conditions of epistemic optimality, in order to explain the mathematicians' reliability it is sufficient to accept the natural supposition that the conditions under which they actually operate, at least in the most fortunate cases, are good enough for the opinion they deliver to be taken to constitute, and a fortiori trivially to track, arithmetical truths. 
In what follows my principal aim will be to argue against the main premise in Divers and Miller's proposal, namely the judgment-dependent nature of arithmetical truth, and so to dispel the appearance of a cheap epistemological vindication of the specific branch of Platonism they offer, under the heading of Non-Gödelian Platonism. However, the specific reasons I shall propose against the characterization of arithmetical truth as judgment-dependent will also raise more general worries concerning the very principle JD-T, namely the potential inconsistency, for every conceivable discourse, between the satisfaction of the substantiality condition and of the independence condition. Therefore, in the final part of the paper, I shall address the very JD-T, and try to indicate under what conditions - remarkably not satisfied in the arithmetical case - a discourse can consistently be taken to sustain it.

\section{Field's Dilemma, and Non-Gödelian Platonism}

In order to illustrate the dilemma proposed by Field, let's start with a rather uncontroversial characterization of Arithmetical Platonism. According to a common presentation, the position endorsed by the Platonist is the semantic interpretation of arithmetical discourse according to which arithmetical statements are true or false of a mind-independent and abstract realm of entities - numbers-which have no spatial and temporal collocation.

Now, consider the following schema:

(1) If mathematicians accept that $p$, then $p$.

With any arithmetical statement instead of $p$, both the Platonist and her opponent accept that (1) is at least in the majority of the cases true. However, how can the Platonist explain (1)? Certainly she "cannot explain the mathematicians beliefs and utterances on the basis of the arithmetical facts being causally involved in the production of those beliefs and utterances" (Field 1989, 230), because arithmetical objects, according to her, are abstract, therefore causally ineffective. Nor is it open to the Platonist to "essay an explanation of mathematical reliability according to which the 'relation' between mathematical thoughts and mathematical truth is constitutive rather than causal. The idea would be that, rather than detect the constitutively independent mathematical facts, mathematicians' (and perhaps others') relevant beliefs actually constitute these facts" (Divers and 
Miller 1999, 280). Such an explanation, it could be argued, would be in tension with the mind-independence of mathematical objects, a trait that is no less vital for Platonism than it is their abstract nature. In the absence of any other proposal as to how (1) could be explained, the alternatives so far reviewed can be assumed to exhaust the logical space. Therefore, if Field is right, no alternative is open to the Platonist. But is Field right?

Not quite, is Divers and Miller's answer. To begin with, they address the question about the proper meaning to be given to the Platonist's contention that mathematical entities exist independently of the mind. According to the authors, a claim to the effect that a given entity exists mind-independently has to be analyzed along the following lines. First, concerning the implicated notion of existence, they endorse Wright's Syntax Priority Thesis (SPT), and maintain that the existence of any entity $\mathrm{E}$ is guaranteed by the truth of any statement featuring an expression to which, by surface syntactic criteria, it is sensible to attribute the function of a singular term, purporting to refer to E; since they endorse the twofold presupposition that (i) at least some arithmetical statements are true, and (ii) that they have the appearance of implicating singular terms-numerals-purporting to refer to numbers, they then conclude that numbers exist ${ }^{6}$. Secondly, as to the notion of mind-independent existence, they say that an object exists in this way if and only if the truth of any statement involving (syntactically appreciable) singular reference to it is not constrained by the presence of minds. In other words, they maintain that for any $p$ of the indicated kind, involving a singular term which purports to refer to $\mathrm{E}$,

(2) E exist mind-independently iff $\diamond$ (it is true that $p \wedge$ there are no minds).

To the extent to which the latter principle is taken to explicate the notion of mind-independent existence endorsed by the Platonist, Field's dilemma then becomes a challenge to explicating the mathematicians' reliability consistently with it. Seen under this perspective, though, its second horn begins to look more promising. Here is Divers and Miller's suggestion.

Suppose you are willing to give content to the metaphor of fact-constitution, mentioned within the second horn of Field's dilemma, by adverting

6. For both a proper statement of the Syntax Priority Thesis, and its application to the mathematical case see Wright (1983, 6 ff.) 
to Wright's principle J-DT. If you also maintain that the correct interpretation of the following bi-conditional, for $p$ ranging over arithmetical statements,

(3) $p \leftrightarrow(\mathrm{C} \rightarrow \mathrm{J} p)$,

is the Euthyphronist's, you are then allowed to maintain that arithmetical truth is, in the sense just advertised, judgment-dependent. Not unexpectedly, this puts you in a position to explain the mathematicians' reliability. If arithmetical truth is judgment-dependent, the explanation why mathematicians tend to have things right just requires the reasonable suggestion that, at least in the most favourable cases, they already operate in epistemic conditions that are sufficiently close to the optimal ones to guarantee the coincidence of arithmetical opinion and arithmetical truth. More unexpectedly, though, you also achieve a position from which the consistency of the latter explanation with (2) above can be easily vindicated. The Syntax Priority Thesis and (2) together motivate the thought that an entity exists independently of the mind just provided that a statement involving syntactically appreciable singular reference to it is possibly true in worlds where there are no minds. The consistency of the judgment-dependent nature of arithmetical truth with the mind-independence nature of mathematical entities therefore just requires that arithmetical statements may be (judgment-dependently) true in worlds where no minds exist; and there seem to be no principled reason why they should not: in order for an arithmetical statement $p$ to be (judgment-dependently) true in a world $w$ where no mind exists, it seems sufficient that $w$ is such as to satisfy the counterfactual condition according to which, were mathematicians to exist and to judge in conditions of epistemic optimality, they would judge that $p$. Provided that a suitable explanation of the advertised counterfactual convergence in opinion of the mathematicians operating in conditions of epistemic optimality is available, then, no potential inconsistency seems to threaten the duo judgment-dependent truth/mind-independent existence (as to what is implicated by the request of such an explanation, see the final sections of this paper). Accordingly, there seems to be no principled difficulty in the proposal according to which Field's challenge can be met by explaining the mathematicians' reliability, on condition that arithmetical truth is correctly characterized as judgment-dependent in nature.

Is, however, the latter characterization correct? The next few sections will be devoted to assessing the arguments which Divers and Miller present 
to vindicate this claim, and to arguing, much in the opposite direction, that it should not be accepted.

\section{Arithmetical truth as judgment-dependent truth?}

As seen in the introductory section, the claim that arithmetical truth is judgment dependent in Wright's sense commits one to showing that there is a substantial and independent specification of the C-conditions mentioned by the following bi-conditional, where $p$ ranges over arithmetical statements,

(3) $p \leftrightarrow(\mathrm{C} \rightarrow \mathrm{J} p)$,

which makes it necessarily and a priori true; moreover, it commits one to showing that the advertised modal and epistemological features of (3) cannot be explained by mentioning the special nature of the implicated truth-conferring (arithmetical) states-of-affairs. In what follows, I set forth to scrutinize Divers and Miller's argument to the effect that there is such specification. As I will try to show, Divers and Miller's identification of the relevant $\mathrm{C}$ conditions seems to raise two central worries: the first one concerns the very truth of (3); the second one is relative to the simultaneous satisfaction of the substantial and independent condition.

Let us begin by inspecting the "constructive epistemology" which motivates Divers and Miller's contention that the C-conditions they specify in the arithmetical case indeed respect the substantiality condition.

Divers and Miller are rather explicit in declaring their strategy:

The accuracy of a subject's judgment is secured en passant by the core condition of conceptual competence [...]. The crucial and advantageous feature of the arithmetical case is that (maximal) conceptual competence alone suffices for accuracy of judgment so long as arithmetic is conceived as knowable a priori and, indeed, as analytic (Divers and Miller 1999, 296).

The suggestion seems to be twofold. On the one hand, it seems to incorporate a general epistemological principle to the effect that

(4) If $p$ is analytic, then $p$ is knowable a priori by exercising one's mastery of the constituent concepts of $p$. 
Naturally enough, (4) suggests that

$\left(4^{*}\right)$ if $p$ is analytic, maximal conceptual competence with the constituents of $p$ is sufficient to make the best judgment as to whether $p^{7}$.

By the second contention,

(5) Arithmetical truths are analytic,

(4) and $\left(4^{*}\right)$ entail that the epistemic conditions $\mathrm{C}$ under which arithmetical opinions are to be counted as best simply require maximal competence with the arithmetical concepts. So, if arithmetical truth is to be regarded as judgment-dependent, it must be the case that the condition of maximal conceptual competence is indeed sustained by a constructive epistemology for mathematics, and that it is an a priori and necessary truth that arithmetical truth varies with opinion delivered in condition of maximal conceptual competence. In what follows I shall advance two major worries against the latter suggestion.

To begin with: if the conditions under which arithmetical opinion is to be regarded as best are conveyed by (4) and $\left(4^{*}\right)$ above, it is doubtful that (3) is as much as true. Just consider Goldbach's Conjecture, and assume, for the sake of argument, that it has a determinate truth-value (if your Dummettian faith doesn't allow you to dare that much, take as an example any currently undecidable sentence which will be decided ${ }^{8}$ ). Suppose further that it is indeed true. Endorsement of (3) commits you to maintaining that were we to address the question whether Goldbach's

7. In its literal interpretation, $\left(4^{*}\right)$ is obviously false. Many further conditions, like reporting and background psychological conditions must be added to make maximal conceptual competence sufficient for best judgment. For instance, the opinions reported by $\mathrm{S}$ under $\mathrm{C}$ must be safe from material self-deception, slip of the tongue, $S$ must be attentive, cognitively lucid, etc. However, such conditions must be arguably included within the specification of the $\mathrm{C}$ conditions for every discourse whose judgment-dependence is under dispute. For the sake of brevity, I just omit reference in $\left(4^{*}\right)$ to such conditions, and concentrate upon the condition whose satisfaction, according to Divers and Miller, is decisive for best judgment in the mathematical case. It must be kept in mind that $\left(4^{*}\right)$ presupposes the satisfaction of the other conditions.

8. I guess that an intuitionist should not find this second request contentious. On the one hand, I agree that a sentence's current undecidability should ban one from endorsing any considered opinion about whether it will be decided. However, on the other hand, even an intuitionist should grant that the actual mathematical practice does deliver good inductive evidence for the belief that at least some currently undecidable sentences will be decided, as mathematicians often prove sentences which before have been undecidable. 
Conjecture is true in conditions of epistemic optimality, we would judge that it is. Yet, the question whether Goldbach's Conjecture is true is one that, right now, we are already addressing, and no one has ever ventured an opinion, as our current situation is not one in which we are justified in believing one way or another. So, it seems to follow that no one has, until now, addressed that very question under the appropriate (optimal) conditions. For an opinion about Goldbach's Conjecture to count as best, under the current proposal, it is however for it to be delivered by someone who is maximally conceptually competent with its ingredient concepts. Therefore, it seems to follow that no one, at the moment, is endowed with a grasp of the relevant concepts which is sufficiently firm. However, this is absurd; there seems to be nothing amiss in our current grasp of the concept of a prime number, the concept of an even number, the concept of a sum, etc. So, either we renounce the idea that having maximal conceptual competence is what makes arithmetical opinion best, or we retain that idea, and then renounce the claim that opinion delivered in optimal epistemic conditions varies with truth. To the extent to which no alternative conception of the C-conditions is proposed, one would then seem to be forced to choose the second alternative, and then to drop (3).

An important moral to be drawn from the argument above, arguably, is about why (4) and $\left(4^{*}\right)$ above do not suffice to vindicate (3). The contrary impression might stem from the nowadays widespread opinion that the notion of analyticity, if it has to play any respectable philosophical role, must be interpreted epistemically. This view, although already present in M. Schlick's reflections on the nature of a priori knowledge, has most recently been rehearsed by P. Boghossian (1996). Boghossian contrasts what he calls the "metaphysical conception" to the "epistemic conception" of analyticity. Roughly, a sentence is metaphysically analytic if the mere fact that the sentence means what it does makes it the case that what the sentence says is true. In turn, a sentence is epistemically analytic if knowledge of its meaning suffices for attaining knowledge to the effect that what it expresses is indeed true. This is not the place to rehearse the arguments Boghossian presents against the metaphysical conception. For what matters, I find them very persuasive. What here matters is that these arguments are also supposed to pave the way to the epistemic conception, and to that extent I don't agree they succeed. The reason, already surfacing in the argument above, has been clearly stated for the first time by W. Künne in a critical review of the different conceptions of analyticity emerging from Schlick's work. Among the other things, Künne discusses 
Schlick's claim that all analytic statements are trivial, namely such that whoever understands them cannot fail to accept them as true. Künne's criticism of Schlick's thesis is that:

we should be saying something correct, when maintaining that a person does not understand, for instance, a tautological statement $S$, even if this person understands each part of $S$ and masters the syntax of the language to which $S$ belongs, and even if he knows how to ascertain whether it is in fact a tautology; it seems clear that someone who still ignored the truth-value of $S$ could well satisfy these conditions. Such use of the word "understand" would certainly be an abuse (my translation, 1982, 214).

Künne's point, simply enough, is that, in many cases, knowledge of a logical or of an arithmetical sentence is mediated by proofs. To the extent to which knowledge of the meaning of their constituting expressions, and of the way they are put together, is consistent with one's inability to figure out a proof, and to the extent to which logical and arithmetical sentences are regarded as paradigm examples of analyticities, knowledge of the meaning of the constituting expressions of an analytic sentence, and of the way they are put together, is not in general guaranteed to yield knowledge. Mutatis mutandis, Künne's criticism of Schlick's characterization of analytic sentences as ones expressing trivialities applies to Boghossian's conception of epistemic analyticity. Accordingly, the reason why Divers and Miller's vindication of (3) fails, at bottom, is the failure of Boghossian's notion of epistemic analyticity?

9. There is, of course, a more charitable reading of Boghossian's proposal which allows preserving epistemic analyticty in the face of the failure of (3). According to a more modest interpretation, Boghossian might be taken to say that whenever a sentence is analytic, one's justification for believing what it says-were one to be justified —will entirely derive from one's understanding of its constituting expressions, and the way in which they are put together. Along with this reading, one's understanding of an analytic sentence is not guaranteed to yield justification for believing what it says; the guarantee we have is that if one is justified in believing an analytic sentence, then the materials upon which this justification supervenes enterly derive from one's understanding of the sentence; so a person's understanding an analytic sentence in this (more modest) sense is compatible with her failing to be justified in believing what it says. This modest reading is clearly unserviceable to the non-Gödelian Platonist, though: what she needs is a principle which guarantees maximal conceptual competence invariably to result in knowledge, and only epistemic analyticity in the stronger reading seems to deliver one which is fit to this role. Moreover, Boghossian also maintains that whoever understands an analytic sentence, although possibly failing to be justified in believing what it says, must be credited with an entitlement (much in T. Burge's sense) to believe what it says, namely a distinctively epistemic right to accept the sentence. So long as one is not disposed to say that we currently are (or at least that some mathematician currently is) entitled to believe (or to disbelieve) Goldbach's conjecture, also the modest reading then seems to face its own problems. 
The latter contention is, admittedly, rather bold. So, it seems advisable to let the success of the strategy I am pursuing be as much as independent from it. The remainder of this section is then designed to offer one additional argument which does not rest on the rejection of the notion of epistemic analyticity.

Let us then suppose, for the argument's sake, that for an arithmetical sentence to be analytic it is for it to be epistemically analytic. If we make this supposition, then (3) is much more plausible under the specification of the relevant $C$ conditions conveyed by $(4)$ and $\left(4^{*}\right)$. In this case, however, the crucial question is about whether, so specified, the $\mathrm{C}$ conditions meet the Substantiality and Independence conditions. What follows is an argument to the effect that they don't.

Before getting started, however, once again it is necessary to examine Boghossian's notion of epistemic analyticity. This time, however, my aim is not to assess its credibility; rather, it is to identify which other principles should enter the picture in order to explain why, as according to that conception, (maximal) semantic competence is indeed sufficient to yield knowledge.

One explanation of epistemic analyticity, applicable to just one rather specific kind of sentences that could be characterized in this way, might run as follows:

$E_{1}$ A sentence of the form $S$ is $P$ is epistemically analytic whenever " $S$ " is synonymous with " $P$ ", and being knowledgeable of this semantic fact is what partially constitutes one's ability to grasp the meaning of " $S$ ", and of " $P$ ".

If understanding what " $S$ " means involves knowing that its meaning is the same as that of " $P$ ", and vice versa, no one understanding both expressions, and understanding the way in which they're put together, may fail to know that $S$ is $P$. This explanation is too specific, however, in view of the fact that many sentences which one might be willing to characterize as analytic do not exhibit the form just envisaged. Boghossian, to name just one, endorses the widespread opinion that many simple truths of logic perform a meaning-constituting function, and that, for this reason, they should be interpreted as implicit definitions of a fundamentally analytic nature. Explaining the epistemic analyticity of implicit definition should involve rather different materials: for instance one might say that no one understanding an implicit definition can fail to know what it says because 
it says what it says in virtue of the successful stipulation that it expresses a truth. However, by generalizing on the first and the second case of analyticities, one might be tempted to suggest that whatever explanation is given of the epistemic analyticity of a statement, it must at least be consistent with the following, loosely explanatory, principle:

$\mathrm{EA}_{2}$ A sentence $S$ is epistemically analytic whenever its truth is guaranteed by facts relative to its meaning.

$\mathrm{EA}_{2}$ is rather generic; yet it has the virtue of hinting at a possible explanation, to be substantiated by individuating the particular role played by each type of the relevant meaning-facts, of the a priori knowability of epistemically analytic sentences. So long as their truth is guaranteed by facts concerning their meanings, and these facts are open to view for a person who's able to understand those sentences, no mystery seems anymore to attach to the claim that that person will know, a priori, what these sentences say upon understanding them. A different and no less crucial advantage of the view under consideration is that it puts no substantive constraint as to what analytic truth is to consist in. Since the principle just trades on meaning-facts guaranteeing a sentence to express a truth, $\mathrm{EA}_{2}$ does not beg the question against the proponent of the "metaphysical conception", according to which meaning-facts determine truth, and is at the same time compatible with the claim that analytic statements, not differently than synthetic sentences, if true are true of reality ${ }^{10}$.

The problem, for Divers and Miller, is that $\mathrm{EA}_{2}$, despite its overt generality, seems already enough to cast doubt on either the Independence, or the Substantiality of the conditions of epistemic optimality that supposedly constrain arithmetical best opinion, and truth. Let's explain by firstly assessing Independence. Let us call MF the meaning-fact(s) which, according to the proponent of (5) (i.e., under the current assumption, of the epistemic analyticity of mathematics), guarantee(s) that a given arithmetical sentence, say $p$, indeed expresses a truth. The overall picture we have described so far allows us to understand why a subject $S$, if maximally competent with the concepts involved in $p$, will then be best suited to ascertain that it expresses a truth: because her conceptual abilities will

10. If a sentence is analytic if its meaning what it does makes it the case that what it says is true, a fortiori its meaning what it does guarantees that it expresses a truth; on the other hand, if a sentence's meaning what it does merely guarantees that it expresses a truth, its truth may well be determined otherwise. 
put her in a position of maximal sensitivity, as it were, with respect to the relevant MF, to which the sentence owes the guarantee of its truth. However, this picture makes implicit reference to a parameter with respect to which arithmetical best opinion must defer, namely the nature of the relevant MF, which therefore is to be accorded a determination which is conceptually prior to, and then unable to be explained in light of the deliverances of best opinion. If so, however, no room seems to be left for construing the activity of a mathematician operating in condition of maximal conceptual competence as one of constituting those truths; rather, the view that such an activity must be construed as one of detecting those truths seems to be inescapable.

Obviously enough, Divers and Miller can be credited, at this point, with one promising reply. However, as I shall show in a moment, it helps in escaping the charge of infringing Independence only at the condition of infringing, in turn, Substantiality. On behalf of Divers and Miller, it might be contended that so long as it is recognized that arithmetical best opinion, by determining the extension of arithmetical truth, is by the same token determining the nature of relevant meaning-facts, there is no difficulty in characterizing arithmetical truth as analytic. For if analytic truth is constrained in the way already envisaged by the relevant meaningfacts, and these facts are best-opinion determined, also analytic truths will be best-opinion determined. So, it might be retorted that the contention that the characterization of arithmetical truth as epistemically analytic is at odds with the satisfaction of the independence condition begs the question of the Euthyphronist concerning (3). For such a contention is based on the outright denial of her central claim, that it is the nature of the relevant conceptual relationships which is constituted by best opinion.

However, such a reply would miss the central point in the foregoing argument. The objection it actually raises is not that analytic truth, simpliciter, requires independently constituted meaning-facts. As the reply correctly emphasizes, we should stay neutral with respect to what determines the nature of mathematical concepts, and the Euthyphronist is primarily in the business of maintaining that it is best opinion that determines it. Rather, the problem is that the characterization of arithmetical truth as epistemically analytic, once paired with the claim that the nature of the relevant conceptual relations is best-opinion determined, cannot be used to motivate the substantial identification of the C-conditions, in the arithmetical case, with maximal conceptual competence. To see why, it is just necessary to appreciate one simple point: if maximal conceptual 
competence is to constrain in any substantive way arithmetical best opinion, it must be possible to assess whether a subject has the advertised feature, independently of what the deliverances of arithmetical best opinion are. However, what the abovementioned reply wants us to consider is precisely the opposite point: along with this reply, whether a subject is maximally competent with the mathematical concepts straightforwardly depends on the deliverances of best opinion, in the very direct sense that whether she has things right, as far as her apprehension of the relations among mathematical concepts goes, depends on what a subject would judge when facing the relevant question in the advertised conditions, because such relationships are determined by what a subject would judge in such conditions. This leads us to the trivial formulation according to which whether subject $S$ delivers best opinion about some arithmetical statement $p$ depends on whether she has things right concerning the relations among the relevant concepts, which in turn now means that it depends on whether she apprehends those relations as they are determined by best opinion. As is clear, circularity - in other words insubstantiality - threatens: we wanted to illuminate the notion of arithmetical best opinion by inquiring under what conditions an opinion may be counted as best, and we are now returned to the answer that we can properly identify those conditions only in terms of the concept-determining role performed by best opinion. The latter point can be made more vivid in light of the following example.

Whether 7 is a prime number, according to the Euthyphronist, depends on whether a subject addressing the question in conditions of epistemic optimality would judge that 7 is a prime number. When asked to specify such conditions, Divers and Miller's Euthyphronist returns the answer that arithmetical truths are analytic, and that, for this very reason, all that is required on a mathematician for her opinion to count as best is that she enjoys maximal conceptual competence with the concept of the number seven, and the concept of a prime number. Undeniably, this is supposed to mean that our mathematician has things right concerning the relations among those concepts; this, in turn, is supposed to compel Divers and Miller's Euthyphronist to say something substantive concerning the conditions under which a subject has things right concerning those concepts. One possibility, which has already surfaced, is that a subject has things right if there is a fact of the matter, concerning whichever number, about whether that number is prime or not, and that the subject has the capability to track such facts. However, this answer is clearly unserviceable for the Euthyphronist, for her primary business 
is to maintain that those facts are constituted, rather than tracked by a maximally competent mathematician. So, she is left with the answer that a subject's competence is maximal when it is in line with the best-opinion determined nature of the concept of a prime number. By endorsing this alternative, however, Divers and Miller's Euthyphronist annihilate the apparent progress made so far; for again, she has to face the question of what the best-opinion determined nature of the concept of a prime number is, and such question can hardly receive a satisfactory answer in the absence of a clear characterization of arithmetical best opinion. So Divers and Miller's Euthyphronist has apparently to accept the consequence that her proposal amounts to clarifying the notion of best opinion through the notion of best opinion: a circle too tight to deserve the title of a substantial characterization.

\section{Explaining counterfactual convergence}

The problem just emphasized seems to exemplify a more general tension between the independence condition and the substantiality condition. If a certain set of conditions has to constrain epistemic optimality in a substantial way-namely, if the supposition that those conditions are satisfied must explain why the opinions formed under those conditions are best suited to track the truth within a given area of discourse- a substantive story has to be told as to why, given the nature of the truths in question, and the means necessary for determining which ones they are, such conditions actually possess this feature. However, when a class of truths is characterized as judgment-dependent, no such story is seemingly forthcoming. For since the truths in questions are constituted rather than detected, the motivation behind any proposed specification of the relevant C-conditions cannot be that, under those conditions, the thinkers are guaranteed to match the truth. Such motivation, making use of the truthmatching metaphor, would in fact demand, in the first place, an independently constituted realm of truths in order for there to be something to be matched in the very first place. This seems to be what goes wrong with Divers and Miller's proposed specification of the C-conditions. Maximal conceptual competence counts as a condition of epistemic optimality only if we conceive of arithmetical truth as independently constituted by the mathematical concepts.

This problem can also be illuminated from a different perspective. 
Take any disputed bi-conditional, like (3) above, expressing the coincidence, within a given area of discourse, between truth and best opinion. Wright's request that the conditions under which an opinion would be best should be specified substantially, at the same time should be taken to entail the request to explaining, substantially, why the opinions are bound to converge in conditions of epistemic optimality. In the very first place, in fact, it could not be true that best opinion, characterized as opinion delivered under a specified set of circumstances $C$, coincide with truth unless it were also true that those opinions, for the very fact of being issued under $\mathrm{C}$, are guaranteed to converge. The problem, for the Euthyphronist, is that the most natural explanation of convergence involves the supposition that those opinions incorporate the aim of representing an independently constituted reality. Let's explain.

Take two devices, like two cameras, which work by receiving inputslight stimulations — and by delivering a given output—a negative film of a given sort. Our inclination to regard the output of a camera as a representation is grounded in our tendency to interpret lack of convergence along the following lines: were we to be faced with two divergent outputs delivered by two different cameras, we would find inescapable the view that either they have been pointed in different directions, or, if they were not, that one of them-or maybe both-functions less than perfectly. In the opposite direction, we take convergence as the hallmark of representational function: when receiving the same inputs and working perfectly, two representational devices cannot but produce the same outputs (Wright 1992, 146).

Clearly enough, this does not logically entail that whenever two devices operating on the basis of the same inputs and working properly cannot but produce converging outputs then they must be thought to be representational devices. Were it so, it would be true as a matter of logic that no discourse-mathematics included-could incorporate a judgmentdependent truth-predicate ${ }^{11}$. Nonetheless, the latter train of thought would

11. The obtaining of the $\mathrm{C}$ conditions arguably guarantees that any two subjects judging that $p$ under those conditions must be receiving the very same inputs, because under those conditions, since by hypothesis such conditions are epistemically optimal, those subjects must enjoy all, and a fortiori the same, (relevant) information: my judgment that $p$ could not be taken to be issued in conditions of epistemic optimality if, under those conditions, some bit of information relevant as to whether $p$ could still escape me. Moreover, it seems to guarantee that they are not the resultant of what could be properly regarded as a cognitive mistake: I couldn't be judging under epistemically optimal conditions if I were in a condition in which a cognitive shortcoming on my part still constituted a possibility. Finally, the truth of the bi-conditional encapsulating the co-variation of truth and best opinion requires convergence. Were the latter, 
seem to motivate at least some pessimism about the very availability of any explanation of convergence alternative to representational function. Whenever a discourse $\mathrm{D}$ is up for a Euthyphronic interpretation, in fact, the intuition that any two divergent $\mathrm{D}$-opinions will have to be explained either by supposing them to be outcome of divergent inputs, or the outcome of cognitive malfunction, will be imposed on us: in that case, the truth of the relevant instance of EC will command the view that, had both opinions been formed under the C-conditions, they would have had to converge, and so enforce the conclusion that one of them, or both, will have to be explained as the resultant either of partial information (so divergent data) or of less-than-proper cognitive functioning (cognitive shortcoming); this, however, will be germane to supposing, along with the suggestion above, that these opinions will already be compromised with representational function.

This result might legitimately raise the following question: since the envisaged convergence of the opinions issued under $\mathrm{C}$ seems to rule out any sensible explanation having no business with the idea that they perform a genuinely representational function, and the latter function imposes the thought that they are responsive to the independent realm of facts which they have the function to describe, how could the simultaneous satisfaction of the Substantiality condition and of the Independence condition constitute as much as a possibility?

Although the latter question might sound rhetorical, I do not think it is. The simple recipe for having both desiderata — substantially explaining convergence without infringing independence-is just to incorporate representational function, while at the same time "cordoning off" its effect with respect to the constitutional role played by subjectivity in determining the extension of the facts over which opinion in a discourse varies; one way could be the following: the facts which a thinker must be thought to be reliably responsive to, when delivering her verdicts under conditions of epistemic optimality, must be divorced from the facts reported by the truths that are allegedly constituted by those verdicts. To put it bluntly: if one's claim is that the truths in A are judgment-dependent, and, along with the suggestion above, one's commitment is to explaining why the satisfaction of $\mathrm{C}$ entails converging verdicts as to the statements expressible within A, such explanation must somehow let this convergence depend on

together with the sameness of inputs/proper cognitive function, to entail representational function, every discourse subject to the latter bi-conditional would have to be interpreted Socratically. 
the fact that, for some independently constituted facts B, subjects operating under $\mathrm{C}$, when delivering best opinion as to $\mathrm{A}$, are reliably responsive to, and therefore epistemically constrained by, B. A possibility is when the properties dealt with within A supervene on the properties dealt with within B.

Consider the claim that truth in the chromatic discourse is judgmentdependent, and the claim that chromatic properties supervene on physical properties. The former claim commits one to accepting, for every colour ascription, bi-conditionals of the kind exemplified by the following one:

$\left(3^{* *}\right) \quad x$ is red iff $(\mathrm{C} \rightarrow \mathrm{S}$ judges that $x$ is red $)$

For $S$ ranging over actual perceivers, and $\mathrm{C}$ specified in a substantial way, acceptance of $\left(3^{* *}\right)$ commits one to providing an explanation as to why, under conditions $\mathrm{C}$, every perceiver constituted as the actual ones would judge, when faced by $x$, that it is red.

Now consider the latter claim, according to which chromatic properties (CP) supervene on physical properties (PP). This claim makes available the following explanation of the convergence of the verdicts delivered by actual perceivers operating under optimal epistemic conditions. Suppose that $R$ designates the distinctive subjective reaction (the distinctive quale) which actually statistically standard subjects experience when observing, in actually good epistemic and environmental conditions (in brief, under conditions $\mathrm{C}$ ), objects reflecting the light along with certain patterns. Suppose that $R$ is the distinctive perceptual seeming that disposes competent speakers of English, when conditions are knowledgeably of the C-kind, to judge that those objects are red. Suppose further that this distinctive pattern of light reflection, resulting in actually standard subjects experiencing $R$, depend on the PP of the objects. If background conditions are kept invariant, the preceding suppositions enforce the thought that every (linguistically competent) perceiver operating under $\mathrm{C}$, if faced by an object $x$ instantiating some PP of the relevant kind, will experience $R$, and will consequently judge that $x$ is red.

In the case at issue we clearly have both desiderata. On the one hand, convergence is explained by the fact that opinion over the distribution of CP is responsive to the PP things possess, so that, whenever the C-conditions obtain, and we thereby have the guarantee that no malfunctioning is in place, we are assured that actual perceivers will deliver converging verdicts. By the same token, the truth conditions of chromatic 
ascriptions, given the constitutive involvement of the subjective reactions elicited by the perceptual interaction with PP, are still to be characterized as judgment-dependent. The point, subtly enough, is that the supervenience thesis accomplishes the delicate function of imbuing the opinions formed under the C-conditions with the "quantity" of responsiveness to mind-independently constituted facts which suffices to preserve a role for subjectivity in determining the extensions of the relevant predicates without at the same time preventing the desired convergence of opinion from arising.

Now, the question is: can the non-Gödelian Platonist devise a similar strategy to explain the convergence in opinion of the mathematicians operating under conditions C? Arguably, this is an accomplishable task only if the opinions delivered by the mathematicians operating under conditions $\mathrm{C}$ can be shown to be responsive to facts different than the arithmetical ones, in a way, however, which still makes room for the attribution to the mathematicians' subjectivity of a role in determining the arithmetical statements' extension.

In concluding this paper, I would like to stress few points that arguably tell against the availability of a similar strategy to the non-Gödelian Platonist.

The main asymmetry between the colour case and the arithmetical case seems to be that, in the former one, there is one single category of objects to which PP and CP can be attributed, namely the physical objects of everyday experience. In the arithmetical case, on the contrary, we cannot have that just one single category of objects is involved. The reason is that, while the arithmetical truths, taken at face value, are about mathematical objects, the facts that the mathematicians must be thought of as being responsive to must not have as constituents mathematical objects, in that they, on pain of conflicting with the judgment-dependence of arithmetical truth, must not be arithmetical facts. If it is so, the non-Gödelian Platonist is faced by an unpalatable alternative. Either she conceives of the mathematical objects, as contrasted with the objects her opinions are representationally responsive to, as non-existing objects. However, no form of Platonism can be arguably divorced from the claim that mathematical objects exist. More than this, the non-Gödelian Platonist is also banned from choosing this alternative by her endorsement of the Syntax Priority Thesis. So she must admit that the arithmetical opinions formed, as it were, under the pressure of the interaction with the underlying category of facts and objects, are about objects to which existence must be attributed. However, if this 
is the horn the Platonist is likely to choose, it is not clear how she will be able to explain how it is that arithmetical opinions, though prompted by the interaction with the underlying basis, nonetheless concern mathematical objects. The most obvious possibility, to plead for an interpretation of arithmetical discourse as being reducible to the discourse about the underlying basis clearly does not work; for it entails the outright denial of the claim that arithmetical statements must be taken at face value, and that numerals actually refer to numbers.

A possibility, at this point, might be constituted by re-interpreting the implicated relation of supervenience as having different relata than the argument above suggests ${ }^{12}$. The idea, defended above, is that the situation of the colour theorist, aiming to vindicate the judgment-dependent nature of chromatic discourse, and that of the non-Gödelian Platonist, aiming to vindicate the judgment-dependent nature of arithmetical discourse, are crucially asymmetric, in that just the former, and not the latter has plain access to the suggestion that the allegedly judgment-dependent discourse trades with facts which supervene on some underlying and ontologically firmer basis. The most compelling reason that has emerged so far is that the nonGödelian Platonist, unlike the colour theorist, has to look for a basis upon which what supervenes is a given kind of objects, rather than properties. This threatens to make a mystery of the nonnegotiable presupposition that mathematical statements indeed involve reference to numbers. However, it might be suggested, there is no principled reason why the non-Gödelian Platonist should look at the relevant supervenience relation that way. Suppose in fact that she identifies the basis with numerals and other mathematical symbols, plus, perhaps, the rules given for their manipulation; and that she then maintains that the relevant supervenience relation obtains among such phenomena and content-involving mathematical experiences. These experiences undeniably involve apparent reference to numbers; yet the ontological status of these entities seems to be somewhat derivative, as such experiences actually supervene on the perceptual encounter with the real objects, the mathematical symbols. The latter intuition, so the proposal might continue, might be captured by drawing a distinction between two different contexts of appraisals for existence claims: on the one side we have the "ontology room" governed by its own more demanding standards, on the other we have the ordinary street outside the ontology room, where the standards of appraisals are somewhat more relaxed. Statements giving

12. I owe this suggestion to an anonymous referee. 
expressions to the content of experiences, as under the current proposal mathematical content-involving experiences, which supervene on something else, as in the case at issue mathematical symbols, are just true in the ordinary sense, yet literally false when assessed within the ontology room. This, it might be contended, might well be enough for having all the non-Gödelian Platonist needs: supervenience, mathematical truth, and judgment-dependency.

Well, in response it should be observed that, in this way, the nonGödelian Platonist would have far more than she needs, indeed too much, and for two different reasons. In the very first place, the proposal under scrutiny crucially depends on the distinction among different contexts of appraisals: by resorting to it, the non-Gödelian Platonist can coherently maintain that mathematical statements are (sometimes) true, yet preserve a role for subjectivity in determining this; yet in this way what becomes mysterious is what in her position, if anything, does deserve the label "Platonism": everybody accepting the distinction could be moved to appreciate that many content-involving experiences of a supervenient variety must turn out true under the ordinary context of evaluation, although arguably false within the ontology room; so the real disagreement among a consistent Platonist and a consistent anti-Platonist, once the distinction enters the picture, seems to concern how existence statements (or statements which imply those statements) should be evaluated within, and not just outside, the more demanding context. Accordingly, it seems safe to conclude that the distinction at issue obfuscates, rather than vindicates, the distinctive Platonist component that non-Gödelian Platonism is in the business to preserve. A second reason for resisting the suggestion moreover derives from the distinction among judgmentdependent truth, and mind-independent existence, which the reply at issue somewhat tends to obscure. The suggestion under scrutiny is that whenever some content-involving experience about some A supervenes (in the re-interpreted sense just advertised) on some base $\mathrm{B}$, we should have to conclude that claims like "there is an A" is judgment-dependently true, and so true just outside, and not within the ontology room; however, what does seem to follow in the first place, once the (revisionary) supervenience thesis is in place, is that A exists mind-dependently; from this it arguably follows that "A exists" if true is also judgment-dependently true, yet at a cost which a sound Platonist shouldn't be able to afford: a Platonist is primarily in the business to vindicate the existence, as mind-independent entities, of numbers; yet by endorsing the suggestion at issue she should 
have to admit that numbers exist, though qua objects of human awareness, and so mind-dependently (for a extended defence of this claim, see Piazza 2009).

Whether there is some other possibility plausibly constitutes an open question. Unless it is convincingly indicated, however, the provisional conclusion seems to be warranted that the non-Gödelian Platonist is not in a good position for explaining convergence. So, not only does the proposed specification of the $\mathrm{C}$ conditions, based on its characterization as analytic truth, fail to constitute a possibility. Also no other characterization, modelled on the colour case, seems to be open to her.

\section{Conclusion}

In this paper I have tried to dispel the impression that the Non-Gödelian Platonist is indeed in a position to escape Field's dilemma. To show this, I have questioned that arithmetical truth can be characterized as judgmentdependent in the sense championed by Crispin Wright.

To show this I have argued that, if it has to be characterized as judgmentdependent, arithmetical truth cannot be characterized as analytic truth. That would entail the violation of the condition according to which, if a body of truth is judgment-dependent, then its extension cannot be conceived as predetermined with respect to the opinions delivered in optimal epistemic conditions by the relevant subjects. More than this, I have argued that the non-Gödelian Platonist is arguably unable to explain, as required by the supposition that arithmetical truth is judgment-dependent, why actual mathematicians would converge in their opinions under ideal epistemic conditions. As in the case of colour ascriptions, such explanation would require the non-Gödelian Platonist to identify a class of facts, different than the arithmetical ones, but arguably related in some way to them, the responsiveness to which explains the convergence of arithmetical opinion in conditions of epistemic optimality. I have argued that no such identification is consistent with respect to the prima facie semantic datum that arithmetical statements are about numbers. 


\section{REFERENCES}

Boghossian, Paul 1996: "Analyticity Reconsidered”. Nô̂s 30, 360-391.

Divers, John \& Miller, Alexander 1999: "Arithmetical Platonism: Reliability and Judgment Dependence”. Philosophical Studies 95, 277-310.

Dummett, Michael 1973: Frege: Philosophy of Language. London: Duckworth.

Field, Hartry 1989: Realism, Mathematics, and Modality. Oxford: Blackwell.

Goldman, Alvin 1967: "A Causal Theory of Knowledge". Reprinted in: Alvin Goldman 1992: Liaisons. Cambridge, MA.: MIT Press, 69-84.

Khlentzos, Drew 2004: "Semantic Challenges to Realism”. In: Edward N. Zalta (ed.), The Stanford Encyclopedia of Philosophy (Fall 2008 Edition). URL = $<$ http://plato.stanford.edu/archives/fall2008/entries/realism-sem-challenge/>.

Künne, Wolfgang 1982: "Analytizität und Trivialität". Grazer Philosophische Studien 16/17, 207-222.

Maddy, Penelope 1990: Realism in Mathematics. Oxford: Clarendon Press.

Piazza, Tommaso 2009: "Platonism, and Mind-Independent Existence". Grazer Philosophische Studien 78, 159-183.

Vinueza, Adam 2001: "Realism and Mind Independence". Pacific Philosophical Quarterly 82, 51-70.

Wright, Crispin 1983: Frege's Conception of Numbers as Objects. Aberdeen: Aberdeen University Press.

— 1992: Truth and Objectivity. Oxford: Harvard University Press. 